Psychotherapeut 2021 - 66:369-371 https://doi.org/10.1007/s00278-021-00531-4 Angenommen: 21. Juli 2021 Online publiziert: 30. August 2021 ๑) Springer Medizin Verlag GmbH, ein Teil von Springer Nature 2021

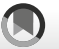

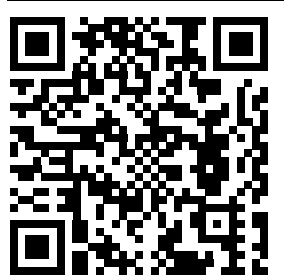

QR-Code scannen \& Beitrag online lesen

\title{
Digitale Interventionen in der Psychotherapie
}

\author{
Antje Gumz ${ }^{1,2}$. Johanna Boettcher \\ 'Psychosomatik und Psychotherapie, Psychologische Hochschule Berlin, Berlin, Deutschland \\ ${ }^{2}$ Klinik für Psychosomatische Medizin und Psychotherapie, Universitätsklinikum Hamburg-Eppendorf, \\ Hamburg, Deutschland \\ ${ }^{3}$ Klinische Psychologie und Psychotherapie, Psychologische Hochschule Berlin, Berlin, Deutschland
}

Der Digitalisierung im Gesundheitswesen wird gesundheitspolitisch hohe Priorität eingeräumt. Entsprechend spielen digitale Anwendungen auch zunehmend eine Rolle im psychotherapeutischen Kontext. Deutschland nimmt in dieser Entwicklung keine Vorreiterstellung ein. Im Gegenteil, es brauchte 20 Jahre intensiver internationaler Forschung, bis erste Formen digitaler Interventionen Einzug in die Versorgung erhielten. Zuerst wagten ausgewählte Kassen, ihren Versicherten digitale Angebote für psychische Probleme zu machen. Seit etwa einem Jahr können Psychotherapeut*innen sog. digitale Gesundheitsanwendungen (DiGA) verschreiben (auch wenn die Auswahl zugelassener Anwendungen noch überschaubar ist).

Die Pandemie hat den Impuls für viele Therapeut*innen gegeben, Videobehandlungen zu erproben. Die Videosprechstunde gewann hiermit sprunghaft an Bedeutung, und die Haltung von Therapeut*innen gegenüber Videosprechstunden war prinzipiell positiv (BeckHiestermann et al. 2021; Connolly et al. 2020; Rabe-Menssen et al. 2020). Viele Therapeut*innen haben ihre Bedenken oder Vorbehalte gegenüber virtuellen Therapien "gezwungenermaßen" aufgegeben, um die Versorgung ihrer Patient*innen zu sichern. Hierin liegt eine große Chance. Wir können nun vorurteilsfreier diskutieren, an welchen Stellen eine Videobehandlung hilft, die psychotherapeutische Versorgung zu verbessern und Versorgungsprobleme zu lösen - wir denken hier beispielsweise an die Unterversorgung auf dem Land oder die Versorgung von Patient*innen, die in ihrer Mobilität eingeschränkt sind oder aus anderen Gründen eine $^{*} n$ Therapeut*in nicht persönlich aufsuchen können. Gleichzeitig sollten wir gerade als Psychotherapeut*innen darauf achtgeben, dass die reale menschliche Begegnung im geschützten Raum einer Praxis nicht grundlos auf ein zweidimensionales virtuelles Miteinander reduziert wird.

In unseren Augen ist dies ein guter Zeitpunkt, einen genauen Blick auf die Vielfalt digitaler Angebote zu werfen und deren Chancen und Grenzen für die ambulante Psychotherapie kritisch zu beleuchten.

\section{Vielfalt digitaler Angebote}

Digitale Anwendungen unterscheiden sich im Hinblick auf ihre Zielgruppe, ihren Platz in der Versorgungskette, ihren Umfang und in ihrem Ausmaß therapeutischer Unterstützung. Es gibt kaum mehr ein Störungsbild, für das noch keine digitale Intervention entwickelt wurde. Dabei sind die Programme auf sehr unterschiedliche Schweregrade psychischer Belastung zugeschnitten. Dies reicht von Symptomen ohne Krankheitswert oder subsyndromalen Belastungen bis hin zu ernsthaften psychischen Störungen. Entsprechend lassen sich die Programme auch an den unterschiedlichsten Punkten der Versorgungskette verorten: Primär-, Sekundärund Tertiärprävention, Überbrückung der Wartezeit auf einen Therapieplatz, Therapie, Nachsorge und Rückfallprophylaxe. So vielfältig die Anwendungsgebiete sind, so vielfältig sind auch die Formen und der Umfang digitaler Interventionen. Zum Teil wird das Internet als reines Informationsmedium genutzt. Hierunter fallen zum einen eher schmale Anwendungen, die 
nur eine Interventionsform umfassen, wie beispielsweise psychoedukative Webseiten oder Monitoring-Apps, zum anderen auch breitere Formen, wie ungeleitete Selbsthilfeprogramme, in denen eine Vielzahl von Interventionen angeboten wird. Die meisten Angebote stammen aus dem Bereich der kognitiven Verhaltenstherapie. In anderen Formen digitaler Interventionen wird das Internet als Kommunikationsmedium genutzt. Hierzu zählen mit einem häufig eher begrenzten Fokus die Chatberatung, aber auch umfängliche Programme der Schreibtherapie sowie auch die Videosprechstunde. Viele Formen digitaler Interventionen kombinieren Informations- und Kommunikationsfunktionen, darunter geleitete Selbsthilfe und manche Formen der "blended therapy", in der digitale Inhalte mit Psychotherapie im direkten Kontakt verknüpft werden.

Das Ausmaß der therapeutischen Begleitung variiert stark nach Form und Umfang der digitalen Intervention. Gänzlich unbegleitete Ansätze stehen Formen begleiteter Selbsthilfe gegenüber, in denen üblicherweise wöchentliche, asynchrone, schriftliche Unterstützung erfolgt. In der Videosprechstunde ist der therapeutische Kontakt, zumindest im Ausmaß, identisch zur Psychotherapie im direkten Kontakt.

\section{Chancen und Grenzen für die ambulante Psychotherapie}

Jede Form der digitalen Intervention hat unterschiedliche Vor- und Nachteile. Kritisch für alle digitalen Interventionen sind Fragen der Datensicherheit. Digitale Interventionen werden häufig als kosteneffizient erachtet, was viele kontroverse Diskussionen ausgelöst hat. Hier ist zu sagen, dass letztlich nur jene Interventionen kosteneffizient sind, bei denen therapeutische Ressourcen gespart werden. Das trifft nur auf ungeleitete Interventionen oder, in einem geringeren Maß, auf geleitete Selbsthilfeinterventionen zu. Auch das Argument, dass Patient*innen durch digitale Angebote Scham und Stigmatisierungsängste überwinden können, greift nur für Interventionsformen ohne oder mit sehr eingeschränktem therapeutischen Kontakt. Umgekehrt ist der häufig formulierte Nachteil, dass digitale Interventionen nur begrenzt ermöglichen, auf akute Krisen zu reagieren, auch nur für die digitalen Interventionen ohne oder mit eingeschränktem therapeutischen Kontakt gültig. Alle Formate, die auch „Face-to-face“(f2f)-Kontakte vorsehen, können ein adäquates Krisenmanagement bieten. Ein großer Vorteil rein digitaler Formate liegt darin, dass sie räumliche und zeitliche Flexibilität bieten. Die meisten digitalen Angebote verbindet, dass sie neue technische Möglichkeiten in der Behandlung psychischer Probleme aufgreifen. Anwendungen auf Smartphones ermöglichen es Patient*innen beispielsweise, ihre Gefühle und Gedanken in schwierigen Situationen direkt festzuhalten und so mögliche Erinnerungseffekte zu minimieren.

\section{Wirksamkeit digitaler Psychotherapie}

Nicht alle Formen digitaler Angebote sind gleich gut belegt. Psychotherapie via Videotelefonie beispielsweise wurde bislang nur sehr wenig untersucht. Begleitete Selbsthilfeprogramme im Bereich der Angst- und Depressionsstörungen hingegen wurden in mehr als 100 randomisierten kontrollierten Studien erfolgreich getestet. Die Effekte im Vergleich zu Kontrollgruppen sind groß (eine kurze Übersicht bieten Andersson et al. 2019; Vergleiche zur f2f-Therapie fassen Carlbring et al. 2018 zusammen). Auch unbegleitete Selbsthilfeangebote wurden vielfach untersucht (zu den Besonderheiten von therapeutischen Apps: Huckvale et al. 2020; Wright et al. 2019). Die Ergebnisse sind jedoch uneindeutiger. Am ehesten lassen sich die Befunde zur Notwendigkeit des Kontakts so zusammenfassen: Therapeutische Unterstützung ist nicht in allen Formen digitaler Interventionen notwendig. Sie hilft jedoch immer, Patient*innen zur Teilnahme zu motivieren, was insbesondere bei weniger attraktiv gestalteten Apps oder schwierigeren Themen und Aufgaben relevant werden kann.

\section{Aktuelle Beiträge zu offenen Fragen}

Während Fragen zur Wirksamkeit zu einem Teil der digitalen Interventionen schon ausreichend beantwortet wurden, sind natür- lich noch viele Fragen offen. Welche weiteren Faktoren sind für das Gelingen einer digitalen Intervention wichtig? Wie wirksam sind digitale Interventionen für besonders vulnerable oder schwer erreichbare Patient*innen? Wie können sie am besten mit bestehenden Versorgungsangeboten verknüpft werden? Welche Rolle spielt die Videosprechstunde? Und natürlich auch, welche Grenzen und Risiken weist diese (nicht mehr ganz neue) Versorgungsform auf? Das vorliegende Sonderheft trägt zu all diesen höchst relevanten Fragen qualitativ hochwertige Beiträge zusammen.

Der besonderen Situation im letzten Jahr Rechnung tragend, widmen sich zunächst gleich drei Beiträge den Erfahrungen mit der Videosprechstunde während der Pandemie. Beck-Hiestermann, Kästner und Gumz berichten die Ergebnisse einer Onlinebefragung unter Psychotherapeut*innen, in der sie untersuchten, wie zufrieden Therapeut*innen mit Videositzungen im Vergleich zur f2f-Therapie waren und inwieweit sich Therapeut*innen unterschiedlicher Verfahren im Hinblick auf ihre Einstellungen zur Videotherapie unterscheiden. Hieran anknüpfend stellen Gumz et al. dar, welche konkreten Vorund Nachteile Therapeut*innen bei der Durchführung von Onlinetherapie erlebten und wie häufig die jeweiligen Vor- und Nachteile benannt wurden. Leukhardt et al. stellen die Ergebnisse einer qualitativen Studie vor, in der sie untersuchten, wie psychodynamische Therapeut*innen und Patient*innen in psychodynamischer Therapie den Wechsel von f2f- zur Videotherapie empfanden.

Um „klassischere" Onlinetherapie, also geleitete und ungeleitete Selbsthilfeinterventionen, geht es in den folgenden Beiträgen. Kreis et al. gehen der Frage nach, inwieweit die bei internetbasierten Interventionen häufig geringen Akzeptanz- und hohen Abbruchraten verbessert werden können. Sie zeigen, dass akzeptanzfördernde Interventionen dazu beitragen können, die Akzeptanz und initiale Nutzung eines transdiagnostischen psychodynamischen Online-Selbsthilfeprogrammes zu verbessern. Domhardt und Kolleg*innen widmen sich der Frage, welche Wirkmechanismen bei digitalen Interventionen zum Tragen kommen. Sie bewegen sich also weg von der Frage, ob 
diese Interventionen wirken und gehen dem, wie sie wirken, nach. Dabei überlegen sie auch, ob es in diesen Formaten eventuell spezifische Wirkmechanismen gibt, die in der traditionellen Therapie nicht greifen.

Zwei weitere Beiträge beschäftigen sich mit bestimmten Patient ${ }^{*}$ innengruppen, die für traditionelle Therapieansätze schwer zu erreichen sind. Wirz und Kolleg*innen beleuchten digitale Interventionen für die besonders vulnerable Gruppe der Geflüchteten, deren Weg in die Gesundheitsversorgung durch sprachliche und andere Barrieren oft holprig ist. Die Autor*innen stellen Apps und Webanwendungen vor, die für Geflüchtete oder deren Behandler*innen aktuell in Deutschland zur Verfügung stehen. Schoenenberg und Martin befassen sich mit einer Patient*innengruppe, die häufig aufgrund starker Schamgefühle keine Behandlung aufsucht. Sie fassen die aktuelle Evidenzlage zum Einsatz von virtueller Realität in der Behandlung von Körperbildstörung zusammen. Sie stellen die Chancen dieser technischen Innovation für die Behandlung von Körperbildstörungen heraus und diskutieren mögliche unerwünschte Effekte.

Um unerwünschte Effekte geht es auch im Beitrag von Baer und Kolleg*innen. Dieser Beitrag widmet sich ganz der "Schattenseite" geleiteter Selbsthilfeinterventionen. Die Autor*innen berichten Verschlechterungs- und Non-Response Raten, sowie quantitative und qualitative Daten zu weiteren Nebenwirkungen. Anhand von zwei Fallbeispielen demonstrieren sie typische Behandlungsschwierigkeiten in diesem Setting.

Die Brücke zur ambulanten Psychotherapie schlägt der Artikel von Bielinski und Berger. Die Autor*innen beschäftigen sich mit der Frage, wie dieser Reichtum an digitalen Interventionen am besten in die bestehende Versorgungslandschaft integriert werden kann. Sie diskutieren verschiedene Formate der Verschmelzung von online und f2f-Therapie und stellen beispielhaft einen Blended-care-Ansatz näher vor. Gerade für niedergelassene Kolleg*innen, die sich mit der neuen DIGAVerschreibungsmöglichkeit konfrontiert sehen, enthält der Artikel wertvolle Hinweise, wie digitale Interventionen zur
Bereicherung unserer psychotherapeutischen Versorgung beitragen können.

So bietet dieses Sonderheft insgesamt viele wertvolle Einblicke in die Vielfalt digitaler Interventionen und entwirft ein differenziertes Bild der aktuellen Lage in Versorgung und Forschung. Wir hoffen, dass das Heft Kliniker*innen und Forscher*innen Impulse zur kritischen Diskussion und Weiterentwicklung digitaler Ansätze geben kann.

\section{Korrespondenzadresse}

\section{Prof. Dr. Antje Gumz}

Am Kölnischen Park 2, 10179 Berlin, Deutschland

a.gumz@phb.de

Interessenkonflikt. A. Gumz und J. Boettcher geben an, dass kein Interessenkonflikt besteht.

\section{Literatur}

Andersson G, Carlbring P, Titov N, Lindefors N (2019) Internet interventions for adults with anxiety and mood disorders: a narrative umbrella review of recent Meta-analyses. Can J Psychiatry 64(7):465-470. https://doi.org/10. 1177/0706743719839381

Beck-Hiestermann FML, Kästner D, Gumz A (2021) Onlinepsychotherapie in Zeiten der CoronaPandemie. Querschnittsbefragung deutscher Psychotherapeuten. https://doi.org/10.1007/ s00278-021-00519-0

Carlbring P, Andersson G, Cuijpers P, Riper H, HedmanLagerlöfE (2018) Internet-based vs. face-to-face cognitive behavior therapy for psychiatric and somatic disorders: an updated systematic review and meta-analysis. Cogn Behav Ther 47(1):1-8

Connolly SL, Miller CJ, Lindsay JA, Bauer MS (2020) A systematic review of providers' attitudes toward telemental health via videoconferencing. Clin Psychol Sci Pract. https://doi.org/10.1111/cpsp. 12311

Huckvale K, Nicholas J, Torous J, Larsen ME (2020) Smartphone apps for the treatment of mental health conditions: status and considerations. Curr Opin Psychol. https://doi.org/10.1016/j. copsyc.2020.04.008

Rabe-Menssen C, Ruh M, Dazer A (2020) Psychotherapeutische Videobehandlungen. https:// www.deutschepsychotherapeutenvereinigung. de/index.php?elD $=$ dumpFile $\& t=f \& f=11152 \&$ token=8efba22d7afdbd29ab5f0a824eb29c7 d2aa94b9c. Zugegriffen: April 2021

Wright JH, Owen JJ, Richards D, Eells TD, Richardson T, Brown GK, Barrett M, Rasku MA, Polser G, Thase ME (2019) Computer-assisted cognitivebehavior therapy for depression: a systematic review and meta-analysis. J Clin Psychiatry 80(2):18r12188
Hier steht eine Anzeige. Springer 